\title{
Group-based educational intervention for advance care planning in primary care: a quasi-experimental study in Japan
}

\author{
Shuji Tsuda ${ }^{1 *}$, Mary R. Janevic ${ }^{1}$, Kota Shikano², Tomoko Matsui ${ }^{3}$, Tsukasa Tsuda ${ }^{4}$
}

\begin{abstract}
Background: Older adults who have health conditions with good prognoses typically fall outside the scope of efforts encouraging advance care planning. We developed group and individual versions of an advance care planning program for use in primary care. Methods: We conducted a quasi-experimental trial in a rural family clinic in Japan. Medically stable patients aged $\geq 65$ years were invited to watch an educational video on advance care planning, followed by an individual $(n=46)$ or group-based $(n=63)$ discussion. Advance directive completion was tracked over four months. Participants completed baseline and follow-up questionnaires and reported occurrence of family discussions about advance care planning and attitudes toward advance care planning. Group discussions were recorded and thematically analyzed to identify barriers and facilitators to engaging in advance care planning. Results: Advance directive completion rates were high for both intervention versions but did not significantly differ between arms $(85.7 \%$ vs. $80.4 \%, p=0.45)$. Only one-fifth of patients in both arms discussed advance care planning with their family after the intervention $(20.7 \%$ and $21.7 \%, p=0.89)$. Patients in the group arm rated their experience slightly higher than those in the individual arm (4.2 and 3.9 out of $5, p=0.023$ ). Qualitative analysis of group discussions revealed that patients were affected by their perceptions of societal norms that prioritize family consensus over patient autonomy; however, these perceptions influenced advance care planning behaviors in inconsistent ways. Conclusions: Group-based advance care planning intervention among medically stable older patients is as effective as an individually-focused discussion in promoting advance directive completion. Future research is needed on ways to enhance patients' ability to discuss advance care planning with their family members. Trial Registration: This study was retrospectively registered on University hospital Medical Information Network Center Clinical Trials Registry (UMIN-CTR) on December 12, 2019 (Trial registry number: UMIN000038916).
\end{abstract}

Keywords: Advance care planning, advance directives, family discussions, older adults, primary care

Department of Health Behavior and Health Education, University of Michigan School of Public Health, USA

Shikano Clinic, JAPAN

Department of Family and Community Medicine, Hamamatsu University School of Medicine, Japan

Kikugawa Family Medicine Center, JAPAN

Corresponding Author*

Shuji Tsuda: 1415 Washington Heights, Ann Arbor, MI 48109, USA

E-mail: tsudas@umich.edu

\section{Contents}

1 Background

2 Methods

2.1 Participants and assignments

2.2 Interventions

2.3 Quantitative data

2.4 Statistical analysis

2.5 Qualitative data

3 Results

3.1 Characteristics of study participants

3.2 Quantitative comparisons of two interventions

3.3 Perceptions regarding ACP behaviors

3.3.1 Facilitators of AD completion

3.3.2 Barriers to AD completion

3.3.3 Facilitators of familial discussions

3.3.4 Barriers to familial discussions

4 Discussion

5 Conclusion

6 List of Abbreviations

7 Declarations

7.1 Ethics approval and consent to participate

7.2 Consent for publication

7.3 Availability of data and materials

7.4 Competing interests

7.5 Funding

7.6 Author's contributions

7.7 Acknowlegements

8

\section{References}

\section{Background}

Advance care planning (ACP) is a process in which adults deliberately prepare for the end of life. This process includes sharing with their significant others their values in life and preferences on medical care that they receive when death is imminent. ${ }^{1}$ These matters affecting death and dying are concerns among older adults regardless of their current health conditions; hence, supporting ACP for all older adults is considered a best practice in contemporary health care. ${ }^{1}$ According to a recent review on ACP practices in palliative care, having a cancer diagnosis and a greater understanding of poor prognosis contributed to increased ACP engagement among patients, but non-cancer patients were less likely to undertake ACP. ${ }^{2}$ This is also true in primary care settings; for example, one review on ACP practices of primary care physicians (PCPs) shows that ACP discussions were held with only $21 \%$ of older patients overall, compared to $69 \%$ in terminal patients and $81 \%$ in patients with mild to moderate dementia. ${ }^{3}$ Thus, older adults who have health conditions with favorable prognoses typically fall outside the scope of efforts encouraging ACP.

Although ACP practices in primary care clinics tend to overlook relatively healthy older patients, evidence suggests that many are nonetheless interested in ACP and 
are waiting for their PCPs to initiate the discussions. ${ }^{4,5}$ Moreover, ACP discussions may contribute to patient satisfaction in physician office visits among older adults. One observational study conducted in a general internal medicine clinic revealed that chronically ill older adults who discussed ACP with their attending physicians were more satisfied with their visit than those who did not. ${ }^{6}$

However, there are various physician- and system-level barriers to implementing ACP practices for these patients. Physician-reported barriers include patients' difficulty understanding treatment options and insufficient time for having such intensive conversations as ACP. ${ }^{7,8}$ Since medically stable older patients are not in pressing need of ACP discussions, these barriers often leave ACP discussions secondary to more immediate clinical consultation topics. Accordingly, promoting ACP for patients in primary care clinics requires programs that will address these barriers.

One potential option for support for ACP in these patients is a program using both video aids and group discussions. ${ }^{9}$ Such a program may overcome the limitations of timeconstrained individual encounters and patient difficulty in envisioning a future health crisis and understanding treatment options. Recently, there has been increased interest in group-based educational programs as a means of improving health outcomes among primary care patients with chronic diseases. These programs, which combine formal instruction from a clinician with support and information from peers, have been shown to increase satisfaction, knowledge, self-efficacy, and healthy behaviors among patients with chronic illness. ${ }^{10,11}$

Group-based educational programs for ACP have been tested in recent studies conducted in the US. They offered older primary care patients video aids and group discussions led by PCPs. ${ }^{9,12,13}$ These programs were associated with improvement in participants' overall satisfaction, ACP knowledge, and completion of advance directives (ADs). ${ }^{9}, 12,13$ However, these studies used uncontrolled pre-post designs and the group programs' effectiveness compared to the more typical individual interventions is unknown.

The primary aim of the present study was to determine whether a video-supported group-format ACP program for older primary care patients with stable medical conditions resulted in a better $\mathrm{AD}$ completion rate and a greater likelihood of familial discussions about ACP compared to an individual session with a physician. As a secondary aim, we used qualitative data to examine the factors that affected decision among group-format participants about whether to engage in familial discussion on ACP and to write ADs.

\section{Methods}

This study is a quasi-experimental clinical trial, in which older patients were assigned to an individual or group-based ACP discussion in a family medicine clinic in a rural area in Japan. The study period was from April 2013 to March
2014. We employed a mixed-method design in which quantitative survey data compared the effectiveness of the two interventions and qualitative data were collected from the group discussions to inform a deeper understanding of the participants' perception of ACP. All trial materials and methods were approved by the Ethics Committee of Hamamatsu University School of Medicine (Registration number: 24-264) and Kikugawa General Hospital.

\subsection{Participants and assignments}

We enrolled adults aged 65 years or older who regularly visited a PCP in the clinic for chronic illness care, had seen the PCP more than three times, and were legally competent. They were invited to the study by their PCPs. Those who had already completed ADs or living wills were ineligible for this study. Those who regularly visited another physician of any specialty were also excluded because of the potential for ACP discussions with this provider. To approximate random assignment, patients who were enrolled in the first half of the month were assigned to the group arm; while those recruited in the second half were to the individual arm. Before the allocation, participants were informed about this study with a leaflet and gave written informed consent.

\subsection{Interventions}

Our intervention consisted of two parts (see Table 1). The first part was an educational session offering a 20-minute video aid and a brochure. The video aid had two components: The first half introduced interviewed patients' stories on $\mathrm{ACP}^{14}$ and explained $\mathrm{ACP}$ and how it can benefit patients and families, while the second half explained each component of the AD and how to complete one. The brochure was designed to review the same information as the second half of the video aid. Immediately after watching the video, the participants attended an assigned discussion, either individual or group. Patients in the individual arm shared their thoughts on ACP with their PCP for about 20 minutes. Patients in the group arm attended a 60-minute group discussion with six to 10 other participants. Groups were facilitated by one of the study investigators who worked as a PCP in the clinic and the discussion addressed preferences for medical care at the end of life and the purpose of ACP. The same two discussion topics were offered in the individual discussions.

Before the research interventions were implemented, we held a 120-minute training workshop for all PCPs and nurses in the clinic. They were asked to attend the workshop since doctors would recruit the participants and engage in individual ACP discussions with them, and nurses would be the person to whom the participants would ask questions and share their concerns when they felt reluctant to do so during the discussion with their doctor. The training workshop provided an opportunity to learn about ACP and to practice discussing ACP with patients through roleplaying. 
Table 1. Contents of ACP Intervention Components

\begin{tabular}{|l|l|}
\hline & \multicolumn{1}{|c|}{ Topics } \\
\hline Video aid & $\begin{array}{l}\text { Conflict frequently faced when engaging in end-of-life decision-making } \\
\text { Consequences of two cases that received life sustaining treatment without known patient wishes } \\
\text { Ideal preparation for end-of-life decision-making } \\
\text { How to write advance directives }\end{array}$ \\
\hline Brochure & Explanation on each component of advance directives \\
\hline Discussion & $\begin{array}{l}\text { Comments on the video } \\
\text { Preference of medical care at the end of life } \\
\text { Purpose of advance directives }\end{array}$ \\
\hline
\end{tabular}

After the interventions, the participants were tracked for a period of time during which two regular visits to their attending PCP were made (bimonthly visiting is standard for stable chronic patients in this clinic). In each followup visit, participants were asked to bring their $\mathrm{AD}$ if they had filled it out beforehand, and their PCP allocated time for answering questions about the ACP and reviewing the completed AD with the participant.

\subsection{Quantitative data}

A self-administered questionnaire was given before the interventions and another at the end of the last follow-up visit, approximately four months after the intervention. The baseline questionnaire collected demographic information including age, gender, educational attainment, perceived health status (poor, fair, good, excellent), hospitalization within the last 5 years, experience of talking about death with others, and knowledge about ADs. This questionnaire also contained seven questions about the participant's beliefs and attitudes about ACP with five response options ranging from strongly disagree to strongly agree. These questions included "I am anxious about participating in the ACP discussion", "I am anxious about writing an AD", "I prefer avoiding caregiving burden on my family", "I am anxious about receiving unwanted care", "I am anxious about pain at the end of life", "I cannot imagine death because it is far ahead", "I am willing to talk about death". For the analysis, responses to these seven questions were dichotomized as "strongly agree" and "agree" meaning "yes."

One of our primary outcomes, completion of ADs, was examined by confirming the scanned document or PCP's statement of AD completion on the participants' electronic medical records. The other primary outcome, an occurrence of family discussion about ACP, was measured with the item "After attending the discussion session, did you discuss your preferred care at the end of life with your family members?" with a dichotomous response option. Additional outcomes were also investigated through the follow-up questionnaire. One of them was a dichotomous question asking "Did your family member attend the discussion session?", and the others were the following questions (strongly disagree to strongly agree): "I was satisfied with the discussion session attended", "I will recommend writing an AD to others", "I will be a proxy for my family member for their ADs."

\subsection{Statistical analysis}

Descriptive statistics were calculated for participants' demographic and health characteristics and beliefs/attitudes toward ACP. For binary outcome variables, intention-totreat analysis was used for chi-square tests and to obtain odds ratios and $95 \%$ confidence intervals for the association between intervention condition (group vs. individual) and the odds of experiencing the outcome. Two-sample t-tests were computed to test for between-group differences in continuous outcome variables. The level of statistical significance was set at $\mathrm{p}<0.05$. These statistical analyses were done with IBM SPSS Statistics v. 23.

\subsection{Qualitative data}

Out of eight group discussion sessions held, four sessions involving 32 participants ( 12 males and 20 females) were recorded and transcribed (average length 56 minutes). An exploratory thematic analysis was conducted by two of the study investigators using Steps for Coding and Theorization. ${ }^{15}$ This method was developed drawing on the analytical procedures of the grounded theory approach and its coding process guides to identify themes and constructs, describe a story-line by incorporating the themes, and develop theories. Our thematic analysis was done with particular attention to the participants' concerns and difficulties when engaging in ACP. The investigators first analyzed the data separately; then compared results with each other and resolved differences in consensus.

\section{Results}

A total of 133 older adults with chronic conditions were eligible for this study and invited to participate. Of them, $24(18 \%)$ declined. The remaining 109 were enrolled, with 63 assigned to the group arm and 46 to the individual arm (Figure 1). After the allocation, three and two participants in the group and individual arms, respectively, did not attend the discussion session, and three and nine, respectively, did not complete the second questionnaire after engaging in their allocated discussion session. Consequently, the numbers of participants who completed the study were 57 and 35 in respective arms (overall attrition rates, 10.5\% and $23.9 \%$ ). 


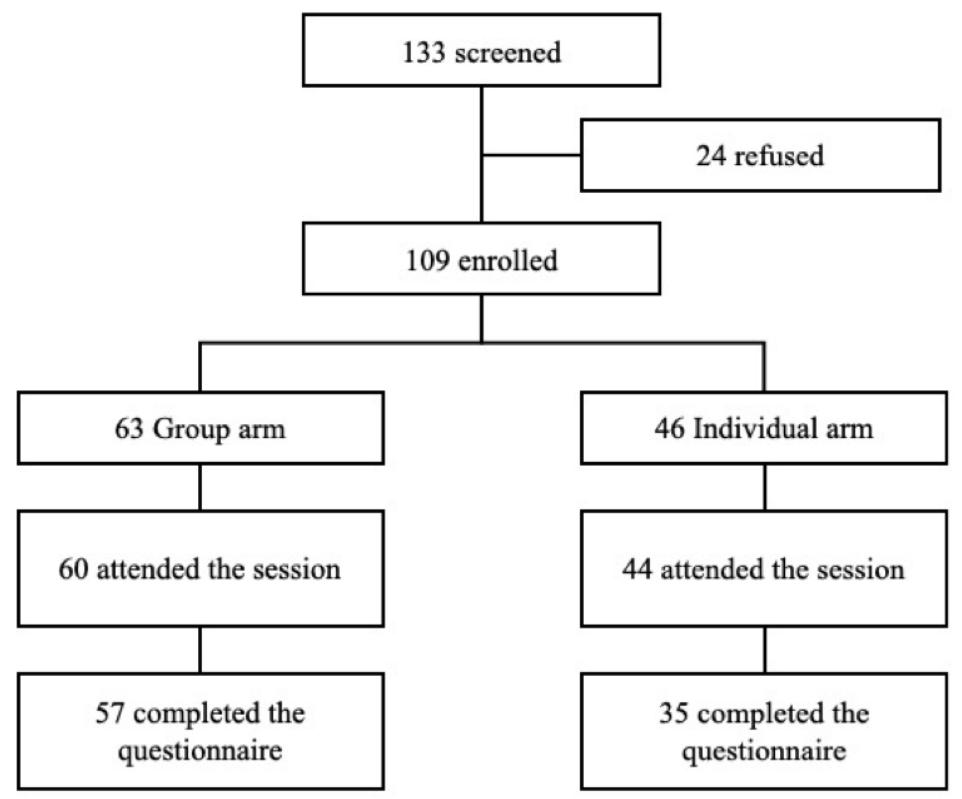

Figure 1. Flow diagram of the study process

\subsection{Characteristics of study participants}

As shown in Table 2, participants mean age was 76.1 in the group arm and 78.8 in the individual arm. Most of them perceived their health status as good or excellent $(88.8 \%$ and $73.9 \%$, respectively). Only $11.5 \%$ and $13.0 \%$ of the participants in each arm were familiar with ADs before the intervention. However, the proportion of participants who had ever talked about death with others was relatively high $(36.1 \%$ and $51.1 \%$, respectively) and half of them indicated that they were willing to talk about death $(58.7 \%$ and $45.6 \%$ ).

Table 2. Patient characteristics and beliefs/attitudes about ACP at baseline $(n=109)$

\begin{tabular}{llllc}
\hline & \multicolumn{2}{c}{ Group arm } & \multicolumn{2}{c}{ Individual arm } \\
& $\mathrm{n}$ & $\%$ & $\mathrm{n}$ & $\%$ \\
\hline Characteristics & & & & \\
Age, years (mean, SD) & 76.1 & 6.8 & 78.8 & 7.8 \\
Gender (female) & 37 & 58.7 & 25 & 54.3 \\
Education (more than high school graduate) & 29 & 46.1 & 24 & 52.2 \\
Health status (good/excellent) & 55 & 88.8 & 34 & 73.9 \\
Experienced hospitalization within last 5 years & 12 & 19.4 & 12 & 26.1 \\
Ever talked about death with others & 22 & 36.1 & 23 & 51.1 \\
Knew what AD is like & 7 & 11.5 & 6 & 13.0 \\
Beliefs and attitudes about ACP* & & & & \\
Anxious about the ACP discussions & 11 & 17.4 & 6 & 13.3 \\
Anxious about writing an AD & 8 & 12.7 & 8 & 17.8 \\
Prefer avoiding caregiving burden on family & 58 & 93.6 & 42 & 91.3 \\
Anxious about receiving unwanted care & 34 & 53.9 & 22 & 48.9 \\
Anxious about pain at the end of life & 36 & 57.1 & 25 & 54.3 \\
Cannot imagine death because it is far ahead & 30 & 47.6 & 25 & 54.4 \\
Willing to talk about death & 37 & 58.7 & 21 & 45.6 \\
\hline
\end{tabular}

ACP, advance care planning; SD, standard deviation; $\mathrm{AD}$, advance directive

*Reported by five response options from 5 strongly agree to 1 strongly disagree, and the figure shows the number of participants who chose strongly agree or agree

3.2 Quantitative comparisons of two interventions After the interventions, AD completion rates were high in both groups, but did not significantly differ between groups $(85.7 \%$ vs. $80.4 \%, p=0.45)$. Only $20.7 \%$ of participants in 
Table 3. Primary and other study outcomes

\begin{tabular}{|c|c|c|c|c|c|c|c|}
\hline & \multicolumn{2}{|c|}{ Group arm } & \multicolumn{2}{|c|}{ Individual arm } & \multirow[b]{2}{*}{$\mathrm{OR} /$ mean $\mathrm{df}^{*}$} & \multirow[b]{2}{*}{$95 \% \mathrm{CI}$} & \multirow[b]{2}{*}{$\mathrm{p}$} \\
\hline & $\mathrm{n} /$ mean & $\% / \mathrm{SD}$ & $\mathrm{n} /$ mean & $\% / \mathrm{SD}$ & & & \\
\hline Familial discussion after the session & 13 & $20.6 \%$ & 10 & $21.7 \%$ & 0.94 & $0.37-2.37$ & 0.89 \\
\hline AD completion & 54 & $85.7 \%$ & 37 & $80.4 \%$ & 1.46 & $0.53-4.02$ & 0.45 \\
\hline Family attendance in the session & 48 & $76.2 \%$ & 29 & $63.0 \%$ & 1.88 & $0.82-4.31$ & 0.14 \\
\hline Satisfied with the session attended $\dagger$ & 4.2 & 0.67 & 3.9 & 0.77 & 0.35 & $0.05-0.66$ & 0.023 \\
\hline Will recommend writing $\mathrm{AD}$ to others $\dagger$ & 2.3 & 0.84 & 2.4 & 0.93 & -0.14 & $-0.52--0.24$ & 0.47 \\
\hline Will be a proxy for my family $\dagger$ & 2.2 & 1.00 & 2.4 & 1.0 & -0.22 & $-0.64-0.19$ & 0.29 \\
\hline
\end{tabular}

$\mathrm{SD}$, standard deviation; $\mathrm{OR}$, odds ratio; $\mathrm{CI}$, confidence interval; $\mathrm{AD}$, advance directive

*Calculated using individual arm as the reference group

$\uparrow$ Reported by five response options from 5 strongly agree to 1 strongly disagree

Table 4. Facilitators and barriers to engaging in family discussions and completing Ads

\begin{tabular}{|l|l|l|}
\hline & \multicolumn{1}{|c|}{ Facilitator } & \multicolumn{1}{c|}{ Barriers } \\
\hline AD completion & $\begin{array}{l}\text { Pursuing an ideal death via AD } \\
\text { Parental responsibilities to the child }\end{array}$ & $\begin{array}{l}\text { Awareness of priority to family's consensual } \\
\text { decision-making over autonomy }\end{array}$ \\
\hline Family discussion & $\begin{array}{l}\text { Awareness of importance of family's role in } \\
\text { decision-making }\end{array}$ & $\begin{array}{l}\text { Awareness of values gap within family } \\
\text { Hesitation to pass on the decision-making } \\
\text { responsibilities to family }\end{array}$ \\
\hline
\end{tabular}

$\mathrm{AD}$, advance directive

the group arm and $21.7 \%$ in the individual arm reported that they engaged in family discussions about their preferred care at the end of life; this rate did not differ between groups $(p=0.89)$ (Table 3). Satisfaction with the discussion sessions was high in both groups, but those in the group arm rated their experience slightly higher than those in the individual arm (4.2 and 3.9 out of 5, p=0.023). Family attendance in the discussion session was more frequent in the group arm $(76.2 \%$ vs. $63.0 \%, p=0.14)$.

\subsection{Perceptions regarding ACP behaviors}

From the group discussions, facilitators and barriers related to completing ADs and engaging in familial discussions are shown in Table 4.

\subsubsection{Facilitators of AD completion}

The study participants regarded ADs primarily as a means to pursue their ideal death, as one of them said "My brothers and other family members expressed their preferences for natural death, and my family chose it. So, likewise, I want my family to do the same thing to me." Also, they stated that completing ADs meant one of the parental responsibilities towards their children. They believed that written ADs will allow death with no futile life-prolongation and will free their children from caregiving burden that might be required when they would become disabled. These beliefs motivated the participants to complete ADs.

\subsubsection{Barriers to AD completion}

In contrast, negative anticipation about how ADs would function in actual decision-making were expressed. The participants were aware of the norm of decision-making practices in their society that a family's consensus was usually prioritized over patient autonomy. For example, one patient described the likely powerlessness of ADs: "Even if the paper (AD) was filled out, it (decision-making) would ultimately depend on the family's preference. It would be done from the sense of values of the family."

\subsubsection{Facilitators of familial discussions}

The same awareness of the family's dominant role in decision-making was reflected in the viewpoint that familial discussions were crucial in ACP. The following quotation is typical of this notion: "My wife will be the person who I expect to take care of me. As such, I should discuss with her before I write it."

\subsubsection{Barriers to familial discussions}

The participants explained that they were willing to have an ACP discussion with family but they often hesitated to start it, especially with younger family members. The participants were aware that it had been their responsibility to take care of their parents and make health-related decisions on behalf of their frail parents, but they wished to avoid pushing the same responsibilities to their children. "Living with the oldest son, who protected his family and home, that person was also expected to take care of his parents, but that was then, and is not true anymore." Some participants were worried that familial discussion would implicitly pass on these responsibilities to their children. "If everything was in fact entrusted to my child, I would feel sorry for him." Others mentioned that they simply did not find cues to initiate the discussion since they were still healthy and independent in life and their family members were preoccupied with busy daily living. 


\section{Discussion}

This quasi-experimental study compared the effectiveness of standardized educational content supplemented by a group vs. individual discussion on completion of ACP for older adults with stable chronic conditions in a primary care setting. We found the same degree of effectiveness in a group discussion as in an individual discussion in producing AD completion and family discussions after the intervention. Qualitative analysis of group discussions revealed that the study participants discussed ADs as a means to pursue their ideal death and to fulfill their responsibilities to their family. Participants were highly affected by expectations of the conventional decision-making practices in the society, that is, family consensus is regarded as more authoritative than patient autonomy. However, this expectation influenced their ACP behaviors in inconsistent ways.

More than $80 \%$ of the study participants in both arms completed ADs following the intervention. In the absence of a specific ACP intervention, Japanese older adults tend not to fill out ADs, as shown in the results of a national survey in 2017 that only $7.8 \%$ of general older adults had completed ADs of any form. ${ }^{16}$ Moreover, the AD completion rate without receiving any interventions among Japanese older patients in primary care clinics was $11.2 \%,{ }^{17}$ which is much lower than findings from a meta-analysis based on data from 2011-16 that $45.6 \%$ of older American adults with any stage of disease completed ADs. ${ }^{18}$ Given these low baseline $\mathrm{AD}$ completion rates among older Japanese adults, it is evident that our interventions effectively increased $\mathrm{AD}$ completion. Moreover, an experimental study in the U.S. testing the effects of group-based ACP programs showed that $\mathrm{AD}$ completion rate after 12-month follow-up was $67 \% .{ }^{12}$ Our ACP intervention utilizing group discussions showed a similar result. Our study provides additional evidence that group-format ACP interventions are an effective way of promoting $\mathrm{AD}$ completion among medically stable older patients. Also, our results imply that support for ACP engagement is insufficient in usual primary care practices in Japan.

During the interventions, we talked about the importance of having family discussions, but only $20 \%$ of the participants in both arms actually held family discussions after the interventions. These percentages are low when compared with the data from a national survey on Japanese perspectives on end-of-life care which showed $46.6 \%$ of general older adults had ever discussed their preferences with their families, friends, or medical providers. ${ }^{16}$ Our study participants, especially those in the group arm, tended to take their family members to the discussion sessions, but this advantage of familial attendance did not translate into greater likelihood of engaging in familial discussions during the follow-up period.

Two recent studies from the U.S. examined the effect of group-visit for supporting ACP for older patients with stable conditions found that the interventions increased the frequency of family discussions and participants' confidence in discussing end-of-life care with their families. ${ }^{9,13}$ These interventions offered two 90-120 minute discussions and provided strategies on how to effectively communicate their preferences with their families. Compared to these interventions, ours did not address these strategies explicitly in the discussion sessions nor in the materials. As a result, our participants may not have learned effective strategies for engaging in family discussions, as was reflected in the lack of discussion about these strategies found in the qualitative analysis.

Family discussion of ACP is crucial since families play an important role when their loved ones face death. A survey in the U.S. revealed $43 \%$ of dying patients required decision-making and $70 \%$ of them lacked decisionmaking capacities. ${ }^{19}$ Additionally, family members who experienced surrogate decision-making often regretted that they had not been informed enough by the patients about their wishes. ${ }^{20}$ From the view of autonomy, families are supposed to advocate for patient wishes, which they become aware of through familial discussions. ${ }^{21}$ However, the intent of familial discussions may be somewhat different in our participants from a rural and, therefore, conservative society in Japan. Qualitative findings indicated that at least some saw family discussions as an opportunity to delegate decision-making to their families.

In Japan, people remain influenced by family-centric beliefs inspired by Confucianism and thus prioritize familycentered decision-making. ${ }^{22,23}$ In this society, patients frequently delegate all decision-making to their families, who decide from their perspectives. ${ }^{24-26}$ Our participants basically believed that family-centeredness was the sense of values shared within their generation, as they had lived their lives fulfilling the responsibilities expected within the family. The qualitative analysis revealed this familycenteredness has two opposite effects on holding family discussions. On one hand, some participants thought that family-centeredness was still present within their family, especially within the older couples. They believed their family will take care of the actual decision-making and, therefore, they considered family discussions necessary. In contrast, others were afraid that family-centeredness would no longer apply to the younger generations and exhibited a great hesitancy to pass on their values and the attendant obligations of family-centeredness to their children. As a result, they were reluctant to hold family discussions with their children during which they might be perceived to be pushing their values and responsibilities onto their family.

In sum, both interventions appeared to be effective at getting people to complete ADs, but less effective at bringing about family discussions. As group-arm participants rated the intervention as slightly more satisfactory and, although not significant, were more likely to bring a family member to the discussion session compared to individual arm, a group-based education program has the potential to produce a better patient experience and more family involvement. 
However, since the occurrence rates of familial discussions for both arms were low, additional content is needed to give people skills and motivation to engage in the family discussion, such as talking in the group about when and how to open familial discussion.

There are several limitations to this study. We did not employ a "no intervention" control group. Also, qualitative data were obtained only from the group arm. Since participants were randomly assigned in a group and each group met only once, contents and topics discussed might have been different from those discussed between a patient and a physician with an established relationship.

Study results may have limited generalizability to other health care systems and different cultures. There were no social workers working in the clinic or available in this study and, thus, the program was basically driven by the physicians, which may have influenced the AD completion rates. Since the program was implemented in one rural clinic in Japan, it was optimized to the given environment in the clinic and the study findings may be unique to the patients influenced by the norms in the society. As was seen in the qualitative findings, influence from societal norms was not negligible when interpreting the results. However, culturally sensitive ACP is a prominent research focus these days and our findings add valuable insight into this topic. $^{26,27}$

\section{Conclusion}

Group-based and individual ACP interventions for older adults with stable chronic conditions are similarly effective at promoting high rates of AD completion. Patients' behaviors related to ACP are affected by their perceptions of societal norms that prioritize family consensus over patient autonomy. Future research is needed to address how to enhance patients' ability to discuss ACP with their family members.

\section{LIST OF ABBREVIATIONS}

ACP: Advance Care Planning

PCP: Primary Care Physician

AD: Advance Directives

\section{DECLARATIONS}

\subsection{Ethics approval and consent to participate}

All trial materials and methods were approved by the Ethics Committee of Hamamatsu University School of Medicine (Registration number: 24-264) and Kikugawa General Hospital.

\subsection{Consent for publication Not applicable}

\subsection{Availability of data and materials}

Derived data supporting the findings of this study are available from the corresponding author on request.

\subsection{Competing interests}

The authors have nothing to disclose.

\subsection{Funding}

This study has no sources of funding.

\subsection{Authors' contributions}

ST, KS, and TT designed and directed the project. ST and KS performed the quantitative analysis. ST and TM performed the qualitative analysis. ST and MJ verified the quantitative and qualitative results and drafted the manuscript. All authors read and approved the final manuscript.

\subsection{Acknowledgements}

The authors thank Dr. Ojima, professor of the Department of Community Health \& Preventive Medicine in Hamamatsu University School of Medicine, for his insightful advice on study design and analysis. We would also like to express our gratitude to the study participants and the providers in the clinic who supported implementing this study.

\section{References}

1. Sudore RL, Lum HD, You JJ, Hanson LC, Meier DE, Pantilat SZ, et al. Defining advance care planning for adults: a consensus definition from a multidisciplinary Delphi panel. J Pain Symptom Manage. 2017;53(5):821-32.e1.

2. Lovell A, Yates P. Advance care planning in palliative care: a systematic literature review of the contextual factors influencing its uptake 2008-2012. Palliat Med. 2014;28(8):1026-35.

3. Glaudemans JJ, Charante EPM Van, Willems DL. Advance care planning in primary care, only for severely ill patients ? A structured review. Fam Pract. 2015;32(1):16-26.

4. Malcomson H, Bisbee S. Perspectives of healthy elders on advance care planning. J Am Acad Nurse Pract. 2009;21(1):18-23.

5. O'Sullivan R, Mailo K, Angeles R, Agarwal G. Advance directives: survey of primary care patients. Can Fam physician. 2015;61(4):3536.

6. Tierney WM, Dexter PR, Gramelspacher GP, Perkins AJ, Zhou XH, Wolinsky FD. The effect of discussions about advance directives on patients' satisfaction with primary care. J Gen Intern Med. 2001;16(1):32-40.

7. Howard M, Bernard C, Klein D, Elston D, Tan A, Slaven M, et al. Survey of health care providers barriers to and enablers of advance care planning with patients in primary care. Can Fam physician. 2018;64(4):e190-8.

8. De Vleminck A, Houttekier D, Pardon K, Deschepper R, Van Audenhove C, Vander Stichele R, et al. Barriers and facilitators for general practitioners to engage in advance care planning: a systematic review. Scand J Prim Health Care. 2013;31(4):215-26.

9. Lum HD, Jones J, Matlock DD, Glasgow RE, Lobo I, Levy CR, et al. Advance care planning meets group medical visits: the feasibility of promoting conversations. Ann Fam Med. 2016;14(2):125-32.

10. Jaber R, Braksmajer A, Trilling JS. Group visits: a qualitative review of current research. J Am Board Fam Med. 2009;19(3):276-90.

11. Kirsh SR, Aron DC, Johnson KD, Santurri LE, Stevenson LD, Jones $\mathrm{KR}$, et al. A realist review of shared medical appointments: How, for whom, and under what circumstances do they work? BMC Health Serv Res. 2017;17(1):113.

12. Lum HD, Sudore RL, Matlock DD, Juarez-Colunga E, Jones J, Nowels $\mathrm{M}$, et al. A group visit initiative improves advance care planning documentation among older adults in primary care. J Am Board Fam Med. 2017;30(4):480-90.

13. Zapata C, Lum HD, Wistar E, Horton C, Sudore RL. Feasibility of a video-based advance care planning website to facilitate group visits among diverse adults from a safety-net health system. J Palliat Med. 2018;21(6):853-6.

14. Nippon Hoso Kyokai (NHK). Ending my life as I wish: a support for fulfilling a person's end of life wishes. https://www.nhk.or.jp/gendai/ articles/3328/1.html. April 3, 2013. (In Japanese). Accessed Sep 23, 
2019.

15. Otani T. "SCAT" a qualitative data analysis method by four-step coding: easy startable and small scale data - applicable process of theorization. Bull Grad Sch Educ Hum Dev. 2008;54:27-44.

16. Ministry of Health Labor and Welfare. National survey on perspectives regarding end of life care. https://www.mhlw.go.jp/toukei/list/d1/ saisyuiryo_a_h29.pdf. Accessed March 1, 2019.

17. Aoki T, Miyashita J, Yamamoto Y, Ikenoue T, Kise M, Fujinuma $\mathrm{Y}$, et al. Patient experience of primary care and advance care planning : a multicentre cross-sectional study in Japan. Fam Pract. 2017;34(2):206-12.

18. Yadav KN, Gabler NB, Cooney E, Kent S, Kim J, Herbst N, et al. Approximately one in three US adults completes any type of advance directive for end-of-life care. Health Aff. 2017;36(7):1244-51.

19. Silveira MJ, Kim SYH, Langa KM. Advance directives and outcomes of surrogate decision making before death. N Engl J Med. 2010;362(13):1211-8.

20. Majesko A, Hong SY, Weissfeld L, White DB. Identifying family members who may struggle in the role of surrogate decision maker. Crit Care Med. 2012;40(8):2281-6.

21. Bravo G, Sene M, Arcand M, Hérault É. Effects of advance care planning on confidence in surrogates' ability to make healthcare decisions consistent with older adults' wishes: findings from a randomized controlled trial. Patient Educ Couns. 2018;101(7):125661.
22. Kwak J, Haley WE. Current research findings on end-of-life decision making among racially or ethnically diverse groups. Gerontologist. 2005;45(5):634-41.

23. Fan R. Self-determination vs. family-determination: two incommensurable principles of autonomy: a report from East Asia. Bioethics. 1997;11(3-4):309-22.

24. Yoshida S, Shiozaki M, Sanjo M, Morita T, Hirai K, Tsuneto S, et al. Practices and evaluations of prognostic disclosure for Japanese cancer patients and their families from the family's point of view. Palliat Support Care. 2013;11(5):383-8.

25. The Japanese Geriatric Society Ethics Committee, Iijima S, Aida N, Ito H, Endo H, Ohrui T, et al. Position statement from the Japan Geriatrics Society 2012: End-of-life care for the elderly. Geriatr Gerontol Int. 2014;14(4):735-9.

26. Menon S, Kars MC, Malhotra C, Campbell AV, Van Delden JJM. Advance care planning in a multicultural family centric community: a qualitative study of health care professionals', patients', and caregivers' perspectives. J Pain Symptom Manage. 2018;56(2):21321.e4.

27. McDermott E, Selman LE. Cultural factors influencing advance care planning in progressive, incurable disease: a systematic review with narrative synthesis. J Pain Symptom Manage. 2018;56(4):613-36. 